Geometry 85 Topology

Volume 6 (2002) 649-655

Published: 14 December 2002

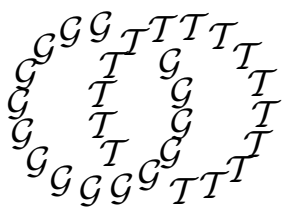

\title{
Convergence groups from subgroups
}

\author{
ERIC L SWENSON \\ Brigham Young University, Mathematics Department \\ 292 TMCB, Provo, UT 84604, USA \\ Email: eric@math.byu.edu
}

\begin{abstract}
We give sufficient conditions for a group of homeomorphisms of a Peano continuum $X$ without cut-points to be a convergence group. The condition is that there is a collection of convergence subgroups whose limit sets "cut up" $X$ in the correct fashion. This is closely related to the result in [7].
\end{abstract}

\section{AMS Classification numbers Primary: 20F32 \\ Secondary: $57 \mathrm{~N} 10$}

Keywords: Group, convergence group, Peano continuum

Proposed: David Gabai

Seconded: Benson Farb, Cameron Gordon
Received: 26 Febrary 2002

Accepted: 15 November 2002

(c) Geometry $8 \mathcal{G}$ Topology $\mathcal{P}$ ublications 
This paper is part of an approach to the weak hyperbolization conjecture in the case where the three manifold, $M$, contains an immersed incompressible surface. The idea of the program is to compactify the universal cover of $M$ with a 2-sphere in such a way that $G=\pi_{1}(M)$ acts on the 2-sphere as a uniform convergence group, and so by Bowditch, [2], $G$ is word hyperbolic.

The application, in this case, is when $X$ is a 2-sphere and the collection $\mathcal{A}$ of subgroups are surface subgroups whose limit sets are circles.

We prove the result in the setting of metric spaces, but it is also true in the more general setting of Hausdorff spaces, and the proof goes through with only minor modifications.

Definition of convergence I We say a group $G$ acting by homeomorphism on a space $X$ acts as a convergence group on $X$ if for each sequence of distinct elements of $G$, there is a subsequence $\left(g_{i}\right)$ and points $p, n \in X$ such that for any compact $C \not \ngtr n$ and any neighborhood $U$ of $p$ there is an $K \in \mathbb{N}$ such that for all $i>K, g_{i}(C) \subset U$. (See figure 1.)

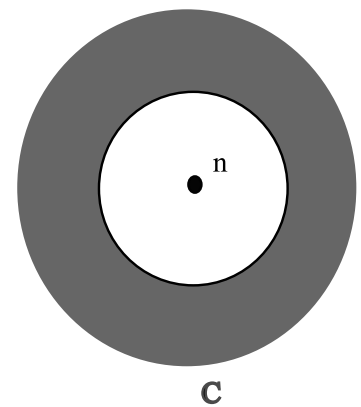

C

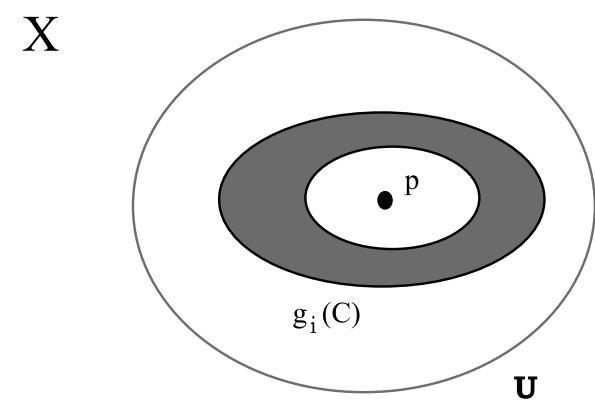

Figure 1

Definition We say that $G$ acts properly on a space $Y$ if for any compact $C \subset Y$ the set $\{g \in G: g(C) \cap C \neq \emptyset\}$ is finite

Remark 1 It is easily shown that $G$ fails to act properly on $Y$ if and only if there exists a sequence $\left(v_{i}\right)$ in $Y$ and a sequence of distinct group elements $\left(g_{i}\right)$ in $G$ such that $v_{i} \rightarrow v \in Y$ and $g_{i}\left(v_{i}\right) \rightarrow \hat{v} \in Y$

Definition of convergence II We say a group $G$ which acts by homeomorphisms on a space $X$ is a convergence group on $X$ if the induced action on the space

$$
\triangle X=\{T \subset X:|T|=3\}
$$


of distinct triples of $X$ is proper. Notice that $\triangle X$ is a quotient of a subset of $X^{3}$ by the action of $S_{3}$ (permuting coordinates), and this defines the topology on $\triangle X$.

In [1], these two definitions are shown to be equivalent in the case where $X$ is compact Hausdorff.

Definition A Peano continuum is a compact connected locally connect metric space. In a continuum $Y$, we say that $A \subset Y$ separates the sets $B, C \subset Y$ if $B$ and $C$ lie in different components of $Y-A$. In a Peano continuum $X$, we say that closed sets $A, B \subset X$ cross if $A \cap B \neq \emptyset, A$ separates points of $B$, or $B$ separates points of $A$.

Definition Let $X$ be a Peano continuum, and $G$ be a group which acts by homeomorphisms on $X$. If $\mathcal{A}$ is a $G$-invariant collection of closed subsets of $X$, we say that the pair $(G, \mathcal{A})$ is a fine pairing on $X$ if the following conditions are satisfied:

(1) $\mathcal{A}$ is cross connected, that is for any $A, B \in \mathcal{A}$ there are $A_{1}, A_{2}, \ldots A_{n} \in$ $\mathcal{A}$ with $A_{1}=A, A_{n}=B$, where $A_{i}$ crosses $A_{i+1}$ for $0<i<n$.

(2) $\mathcal{A}$ is null. That is: For any $\epsilon>0$, the set of elements of $\mathcal{A}$ with diameter at least $\epsilon,\{A \in \mathcal{A}: \operatorname{diam}(A)>\epsilon\}$ is finite.

(3) $\mathcal{A}$ is fine. That is: For any $x, y \in X$ there exists a finite $\mathcal{B} \subset \mathcal{A}$ such that $\cup \mathcal{B}$ separates $x$ from $y$.

We need the following two results from continuum theory.

Lemma 1 If $M$ is a Peano continuum and a closed set $A$ does not separate the closed connected set $B$ from the closed connected set $C$, then there exists a neighborhood $V$ of $A$ which does not separate $B$ from $C$. (We are of course assuming that $A \cap(B \cup C)=\emptyset$.)

Proof For $x, y \in M-A$, define $x \sim y$ if there exist a closed neighborhood $D$ of $A$ which does not separate $x$ from $y$. Clearly $B$ is in a single equivalence class as is $C$. Let $b \in B$. Define $W=\{x \in M-A: x \sim b\}$. Since $W$ is the union of components of open sets of $M$, and $M$ is locally connected, it follows that $W$ is open in $M$. Notice that $\bar{W} \subset W \cup A$ and so $W$ is closed in $M-A$. Thus $W$ is a component of $M-A$ and so $W \supset C$. It follows that there is a closed neighborhood $V \subset M-(B \cup C)$ of $A$ which does not separate some point of $C$ from $b$, and so $V$ does not separate $B$ from $C$. 
Lemma 2 Given $Z$, a Peano continuum, for any $\epsilon>0$ there is $\delta>0$ such that if $A, B$ are crossing closed sets of $X$ with $\operatorname{diam}(A)<\delta$ and $\operatorname{diam}(B)<\delta$, then $\operatorname{diam}(A \cup B)<\epsilon$.

Proof Suppose not, then for some $\epsilon>0$ there are sequencea $\left(A_{n}\right)$ and $\left(B_{n}\right)$ of closed subsets of $X$ with $A_{n}$ crossing $B_{n}$ for each $n, \operatorname{diam}\left(A_{n}\right), \operatorname{diam}\left(B_{n}\right)<$ $\frac{1}{n}$, but $\operatorname{diam}\left(A_{n} \cup B_{n}\right) \geq \epsilon$ for all $n$. Using compactness, we may assume that $A_{n} \rightarrow a \in X$ and $B_{n} \rightarrow b \in X$ which implies that $d(a, b) \geq \epsilon$. Choose disjoint connected neighborhoods $U$ and $V$ of $a$ and $b$ respectively. For all $n>>0$, $A_{n} \subset U$ and $B_{n} \subset V$, which implies that $A_{n}$ and $B_{n}$ do not cross.

Lemma 3 Let $(G, \mathcal{A})$ be a fine pairing on $X$ and $\mathcal{B} \subset \mathcal{A}$ with $\mathcal{B}$ finite and cross connected. If $\left(g_{i}\right)$ is a sequence of elements of $G$ then either $\left(g_{i}(\cup \mathcal{B})\right)$ is null, that is $\forall \epsilon>0, \exists N$ such that $\operatorname{diam}\left(g_{i}(\cup \mathcal{B})\right)<\epsilon$ whenever $i>N$, or there exists $B \in \mathcal{B}, A \in \mathcal{A}$ and a subsequence $\left(g_{i_{n}}\right)$ of $\left(g_{i}\right)$ with $g_{i_{n}}(B)=A$ for all $n$.

Proof We assume that $\left(g_{i}(\cup \mathcal{B})\right)$ is not null. By Lemma 2 there is $B \in \mathcal{B}$ with $\left(g_{i}(B)\right)$ not null. Thus there is $\epsilon>0$ and a subsequence $\left(g_{i_{m}}\right)$ with $\operatorname{diam}\left(g_{i_{m}}(B)\right)>\epsilon$ for all $m$. Since $\mathcal{A}$ is null, the set $\left\{g_{i_{m}}(B)\right\}$ is finite and the result follows.

Main Theorem Let $X$ be a Peano continuum without cut points, and $(G, \mathcal{A})$ is a fine pairing on $X$. If, for each $A \in \mathcal{A}, \operatorname{Stab}(A)$ acts as a convergence group on $X$, then $G$ acts as a convergence group on $X$.

Proof We will show that $G$ acts properly on $\triangle X$. Assume not, then we have a sequence $\left(\mathbf{v}_{i}\right)$ in $\triangle X$ and a sequence of distinct group elements $\left(g_{i}\right)$ in $G$ such that $\mathbf{v}_{i} \rightarrow \mathbf{v} \in \triangle X$ and $g_{i}\left(\mathbf{v}_{i}\right) \rightarrow \mathbf{w} \in \triangle X$. We may assume we have $\mathbf{v}_{i}=\left\{x_{i}^{1}, x_{i}^{2}, x_{i}^{3}\right\}, \mathbf{v}=\left\{x^{1}, x^{2}, x^{3}\right\}$ and $\mathbf{w}=\left\{y^{1}, y^{2}, y^{3}\right\}$ with $x_{i}^{j} \rightarrow x^{j}$ and $g_{i}\left(x_{i}^{j}\right) \rightarrow y^{j}$ for $j=1,2,3$.

For $j=1,2,3$ choose a finite $\mathcal{B}^{j} \subset \mathcal{A}$ with $x^{j} \in V^{j}$, a component of $X-\cup \mathcal{B}^{j}$. Using fineness (and compactness of $X$ ) we can arrange that $\overline{V^{j}} \cap \overline{V^{k}}=\emptyset$ for $j \neq k$. Now choose a finite sequentially connected $\mathcal{B} \subset \mathcal{A}$ so that $\mathcal{B}^{j} \subset \mathcal{B}$ for $j=1,2,3$.

We now choose connected neighborhoods $W^{j}$ of $y^{j}$ for $j=1,2,3$ such that $\overline{W^{j}} \cap \overline{W^{k}}=\emptyset$ for $j \neq k$.

We now show that the sequence $\left(g_{i}(\cup \mathcal{B})\right)$ is null. For if not, then by Lemma 3 we may assume that there is a $B \in \mathcal{B}$ and $A \in \mathcal{A}$ such that $g_{i}(B)=A$ for all 
$i$. Thus $h_{i}=g_{i} g_{1}^{-1} \in \operatorname{Stab}(A)$. Letting $\mathbf{u}_{i}=g_{1}\left(\mathbf{v}_{i}\right)$ we have $h_{i}\left(\mathbf{u}_{i}\right) \rightarrow \mathbf{w}$ and $\mathbf{u}_{i} \rightarrow g_{1}(\mathbf{v})$. By remark 1, this would imply that $\operatorname{Stab}(A)$ didn't act properly on $\triangle X$ which is a contradiction. Thus the sequence $\left(g_{i}(\cup \mathcal{B})\right)$ is null and passing to a subsequence we may assume that $\left(g_{i}(\cup \mathcal{B})\right) \rightarrow z \in X$. (That is to say that for any neighborhood $U$ of $z$, there is an $M$ such that if $i>M$ then $g_{i}(\cup \mathcal{B}) \subset U$.)

Since $\overline{W^{1}}, \overline{W^{2}}$ and $\overline{W^{3}}$ are disjoint, we may assume $z \notin \overline{W^{2}} \cup \overline{W^{3}}$, and so passing to a subsequence we may assume that $g_{i}(\cup \mathcal{B}) \cap W^{2}=\emptyset=g_{i}(\cup \mathcal{B}) \cap W^{3}$. The point $z$ is not a cut point of $X$. By Lemma 1 , for $i \gg 0, g_{i}(\cup \mathcal{B})$ does not separate $W^{2}$ from $W^{3}$. (See figure 2.)

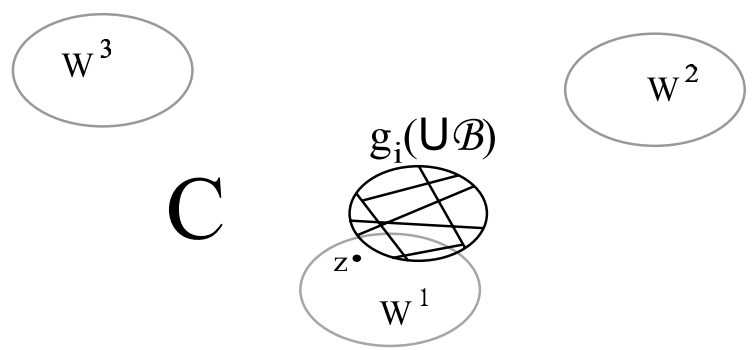

Figure 2

Notice that for $i \gg 0, g_{i}\left(x_{i}^{j}\right) \in g_{i}\left(V^{j}\right) \cap W^{j} \neq \emptyset$.

Thus for such a (very) sufficiently large $i, W^{2} \cup W^{3}$ is contained in a single component $C$ of $X-g_{i}(\cup \mathcal{B})$ and $g_{i}\left(V^{2}\right) \cap W^{2} \neq \emptyset \neq g_{i}\left(V^{3}\right) \cap W^{3}$. This implies that $C \subset g_{i}\left(V^{2}\right)$ and $C \subset g_{i}\left(V^{3}\right)$ contradicting the fact that $V^{2} \cap V^{3}=\emptyset$.

We will show that the theorem is false if the hypothesis that $\mathcal{A}$ is fine is removed.

Example Let $G$ be the Kleinian reflection group generated by reflection in the sides of a regular right-angle hyperbolic dodecahedron. For each reflection $r \in G$ let $P_{r}$ be the plane in $\mathbb{H}^{3}$ fixed by $r$. By standard results, the centralizer of $r$ acts cocompactly on $P_{r}$. Let $H_{r}=\operatorname{Stab}\left(P_{r}\right)=\operatorname{Stab}\left(\partial P_{r}\right)$ where $\partial P_{r}$ is the boundary circle of $P_{r}$ in $S^{2}=\partial \mathbb{H}^{3}$. Since $H_{r}$ acts cocompactly on $P_{r}$, it follows that the limit set $\Lambda H_{r}=\partial P_{r}$. Notice that the Main theorem applies in this setting, as the collection of circles $\mathcal{A}=\left\{\partial P_{r} \mid r \in G\right.$ a reflection $\}$ is fine. In fact this collection of circles gives one of the subdivision rules studied by Cannon, Floyd and Perry [4, pages 16-17].

We now alter this example so that the collection $\mathcal{A}$ is no longer fine, and the action is no longer a convergence action, but all of the other hypothesis of the 
Main theorem apply. By Baire's theorem, there is a point $s \in S^{2}$ such that $s \notin \partial P_{r}$ for every reflection $r \in G$. Choose a convergence sequence of distinct group elements $\left(g_{i}\right) \subset G$ with $n \neq s$ such that for any compact $C \not \nexists n$ and any neighborhood $U$ of $s, g_{i}(C) \subset U$ for all $i>>0$.

Now let $\mathcal{S}$ be the set of translates of $s$ by $G$. For each $t \in \mathcal{S}$, we replace the point $t$ by the circle of directions $\hat{t}$ at $t$ (so $\hat{t}$ is a circle of length $2 \pi$ ). Rigourously let $\hat{\mathcal{S}}=\{\hat{t} \mid t \in \mathcal{S}\}$, and let $X=\left(S^{2}-\mathcal{S}\right) \cup \hat{\mathcal{S}}$. We now describe the topology on $X$ and show that $G$ acts on $X$ via homeomorphisms. There is the obvious "quotient" function $f: X \rightarrow S^{2}$ given by $f(x)=x$ for $x \notin \cup \hat{\mathcal{S}}$ and $f(\hat{t})=\{t\}$ for $t \in \mathcal{S}$. To define the topology on $X$ we describe a neighborhood basis of $X$. For $x \notin \cup \hat{\mathcal{S}}$ and for $U$ a neighborhood of $f(x), f^{-1}(U)$ will be a neighborhood of $x$.

For $x \in \hat{t} \in \hat{\mathcal{S}}$, let $\epsilon \in(0, \pi)$. Let $\alpha$ be the geodesic segment starting at $t$ of length $\epsilon$ in the direction of $x$. Define

$$
U_{\epsilon}=\left\{y \in S^{2}: 0<d(t, y)<\epsilon, \text { and } \angle_{t}(\alpha,[t, y])<\epsilon\right\} .
$$

Now $V_{\epsilon}=f^{-1}\left(U_{\epsilon}\right) \cup(x-\epsilon, x+\epsilon)$, where $(x-\epsilon, x+\epsilon)$ is the open interval of the circle $\hat{t}$. (See figure 3.)
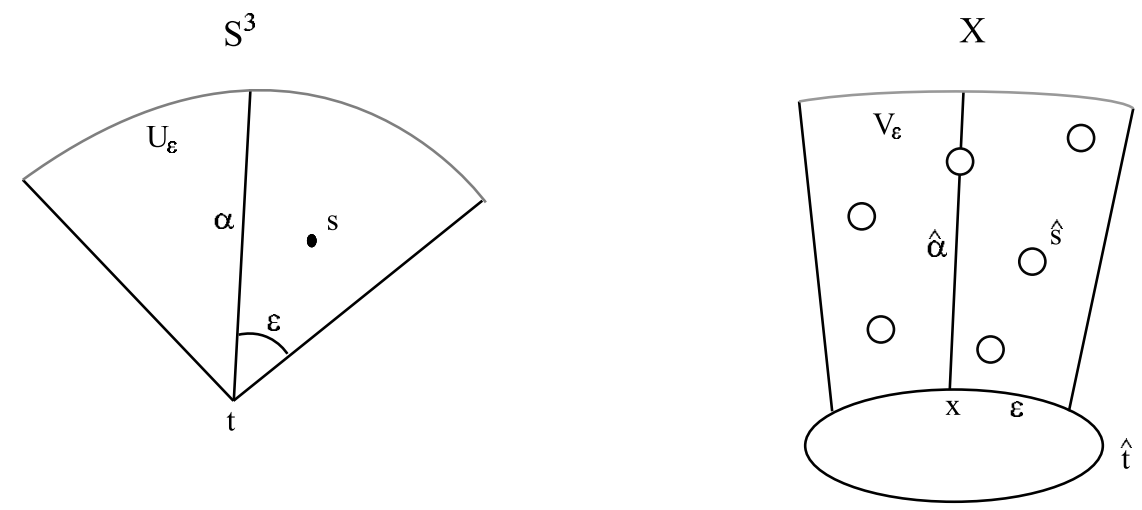

Figure 3

This gives a topology on $X$ which is metrizable since $X$ is regular and has a countable basis ( $\mathcal{S}$ is countable). Since $G$ acts conformally on $S^{2}, G$ preserves angles, and hence the action of $G$ on $S^{2}$ "extends" to an action of $G$ on $X$, and this action commutes with $f$, ie. for $g \in G, g \circ f=f \circ g$.

Now for any reflection $r \in G$, by definition of $s, \mathcal{S} \cap \partial P_{r}=\emptyset$. Thus $\partial P_{r} \subset X$ and it follows that the stabilizer $H_{r}$ acts as a convergence on $X$. 
However, $G$ does not act as a convergence group on $X$, for our original convergence sequence $\left(g_{i}\right)$ will have no convergence subsequence. To see this, let $c \in X$ and take a closed annulus $D \subset S^{2}$ separating $n$ from $s$ with $f(c) \notin D$. Notice that by definition of $\left(g_{i}\right), g_{i}(D) \rightarrow s$. Let $\hat{D}=f^{-1}(D)$, and notice that $\hat{D}$ is compact. Clearly for any subsequence $\left(g_{n_{i}}\right)$ of $\left(g_{i}\right), g_{n_{i}}(\hat{D}) \rightarrow \hat{s}$. Since this is converging to an entire circle, it cannot converge to a single point, and so $\left(g_{n_{i}}\right)$ is not a convergence sequence with repelling point $c$. Thus $G$ does not act as a convergence group on $X$, even though the action of $G$ on $S$ satisfies all of the hypothesis of the main theorem except that $\mathcal{A}$ is not fine on $X$.

We can alter this example slightly by gluing a disk into each of the circles $\hat{t}$, and obtain a new action of $G$ on $S^{2}$ which is not a convergence action (fineness still fails).

\section{References}

[1] B Bowditch, Convergence groups and configuration spaces, from: "Geometric group theory down under (Canberra, 1996)", de Gruyter, Berlin (1999) 23-54

[2] B Bowditch, A topological characterisation of hyperbolic groups, J. Amer. Math. Soc. 11 (1998) 643-667

[3] A Casson, D Jungreis, Convergence groups and Seifert fibered 3-manifolds, Invent. Math. 118 (1994) 441-456

[4] J Cannon, W Floyd, W Perry, On the conformal invariance of tilingsystems, preprint (1996)

[5] David Gabai, Convergence groups are Fuchsian groups, Annals of Mathematics, 136 (1992) 477-510

[6] J Hass, J Rubinstein, P Scott, Compactifying coverings of closed 3manifolds, J. Diff. Geom. 30 (1989) 817-832

[7] E Swenson, Axial pairs and convergence groups on $S^{1}$, Topology 39 (2000) 229-237

[8] P Tukia, Homeomorphic conjugates of Fuchsian groups, J. Reine Angew. Math. 391 (1988) 1-54

[9] P Tukia, Convergence groups and Gromov's metric hyperbolic spaces, New Zealand J. Math. 23 (1994) 11-24 